\title{
ON A NECESSARY CONDITION FOR THE VALIDITY OF THE RIEMANN HYPOTHESIS FOR FUNCTIONS THAT GENERALIZE THE RIEMANN ZETA FUNCTION $\left(^{1}\right)$
}

\author{
BY \\ RONALD ALTER
}

I. Introduction. It is known that the Riemann hypothesis is equivalent to the statement that all zeros of a certain entire function $\Phi(z)$ are real. In 1914, Grommer [6] gave a countable set of conditions which are necessary and sufficient for all the zeros of an entire function to be real. The conditions consist in the positivity of certain determinants of increasing orders, formed with the coefficients of the power series expansion of the entire function under consideration. In 1927, Pólya [9] stated the first Grommer condition as a necessary condition for the validity of the Riemann hypothesis. He also pointed out that there seemed to be no method by which one could verify this condition. (For a statement of this condition see (8) in §II.)

In 1956, Hayman [7] considers functions $f(z)=\sum_{n=0}^{\infty} \alpha_{n} z^{n}$ analytic inside $|z|<R$ $\leq \infty$, and satisfying some additional conditions. For this large class $F$ of entire functions (defined in $\S I$ ), of which $\Phi(z)$ is also an element, Hayman found an asymptotic formula for their coefficients. This asymptotic estimate generalizes Stirling's formula: $1 / n ! \sim(e / n)^{n}(2 \pi n)^{-1 / 2}$, to which it reduces in the case $f(z)=e^{z}$. However, the situation with respect to the Grommer conditions did not change because the error term introduced by the use of Hayman's formula is of the same order as the determinants to be computed.

By considering functions of a smaller class $F_{1} \subset F$, Grosswald [5] strengthened Hayman's assumptions. This enabled him to generalize Hayman's formula by obtaining an asymptotic series for the coefficients $\alpha_{n}$ of the class of functions $F_{1}$ which corresponds to the asymptotic series for the $\Gamma$-function to which it reduces if $f(z)=e^{z}$. (Grosswald's result is stated in $\S I I$ as Theorem 1.) Using such asymptotic series for the coefficients of $\Phi(z)$, Grosswald shows that the first of Grommer's conditions is satisfied for all sufficiently large coefficients of $\Phi(z)$. The method used is constructive, thus it is theoretically possible to determine an upper bound for the finitely many coefficients not covered by the main result and by direct computation show that they satisfy Grommer's first condition. Hence Grosswald has shown that a certain condition $\mathrm{C}$, necessary, but by no means sufficient, for the validity of the Riemann hypothesis is indeed satisfied.

Received by the editors May 6, 1966.

(1) This work is based on a doctoral dissertation that was submitted to the University of Pennsylvania. 
In this paper a class $F_{2}$ of functions depending on several variable parameters is studied. ( $F_{2} \subset F$ which also satisfies (13).) It is shown, in §III, after the proof of Theorem 5 , that for all values of the parameters belonging to certain ranges $F_{2} \subset F_{1}$. The major result of $\S I I I$ is Theorem 10, most of the other theorems in this section are proven in order to establish Theorem 10. Theorem 10 establishes that all functions in $F_{2}$ satisfy (9); hence it follows that the method used to verify the positivity of the determinants, corresponding to the problem of the Riemann zeta function $\zeta(s)$, works equally well for all other functions of the class $F_{2}$. Thus all of these functions satisfy a "condition $C$ " necessary for the validity of a "Riemann hypothesis."

Although Theorems 2-9 of §III are primarily, as stated in the above paragraph, to establish Theorem 10 these results do have some independent interest. In particular Theorems 2 and 3 give us information about the functions defined in (11), (11') and (12). Theorem 4 estimates the size of the $k$ power of a linear operator operating on the logarithm of a Dirichlet series. Theorems 5, 6 and 9 give us additional information about $F_{2}$ functions and their coefficients. In Theorem 7 we have a very particular result about $\Gamma$-functions and finally Theorem 8 gives us information about a root $r$ of the transcendental equation (1).

There are many functions which generalize the Riemann zeta function, and each of them has a Riemann hypothesis that is associated with it. In this paper it is also shown that, for particular admissible values of the variable parameters, many of these classical functions which generalize the Riemann zeta function belong to class $F_{2}$. It thus follows that all these functions satisfy a corresponding necessary condition $\mathrm{C}$ for the validity of their Riemann hypothesis.

Among the functions studied are $L$-functions of real, primitive characters, Ramanujan's function $Z(s, \tau)=\sum_{n=1}^{\infty} \tau(n) / n^{s}$ where $\tau(n)$ is the arithmetic function defined by

$$
x\left\{\prod_{1}^{\infty}\left(1-x^{m}\right)\right\}^{24}=\sum_{1}^{\infty} \tau(n) x^{n}, \quad|x|<1,
$$

and Dedekind's zeta function $\zeta(s, K)=\sum_{\mathfrak{a} \subset K}(\mathrm{Na})^{-s}$ of a field $K$ of algebraic numbers. In order to show that condition $C$ is necessary but not sufficient the Epstein zeta function $\mathrm{Z}(s, Q)$, for certain values of the discriminant of the quadratic form $Q(x, y)$, is also studied. It is shown that $\mathrm{Z}(s, Q)$ does indeed satisfy condition $\mathrm{C}$ although it has two real zeros in the interval $(0,1)$. (For a proof that the Riemann hypothesis is false see [3], in particular p. 367). Finally, for completeness, it is shown by an example, that not all functions of class $F$, in fact not all functions of class $F_{1}$, satisfy condition $\mathrm{C}$.

Throughout it should be kept in mind that all results obtained in the paper refer only to $n$ sufficiently large.

\section{Notation and preliminary material.}

2. Let $f(z)=\sum_{n=0}^{\infty} \mathfrak{a}_{n} z^{n}$ be analytic inside the circle $|z|<R(\leq \infty)$, real on the real axis and such that $\lim _{x \rightarrow R} f(x)=+\infty$. 
Set $a_{1}(z)=z d(\log f(z)) / d z=z f^{\prime}(z) / f(z)$, we define inductively for $\nu>1, a_{v}$ $=z d a_{v-1}(z) / d z$.

Let $A=$ the class of real-valued functions, $a(x)$, such that for $\nu \geq 3$ we have that $a_{v}(x) \leqq a(x)$, for sufficiently large $x$, which may depend on $\nu$.

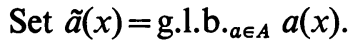

We assume there exists a $\delta(x)$ satisfying, for some $a \in A$,

(i) $\lim _{x \rightarrow R} \delta^{2}(x) a_{2}(x)=\infty$,

(ii) $\lim _{x \rightarrow R} \delta^{3}(x) a(x)=0$,

(iii) $\lim _{x \rightarrow R} \lambda(x, \delta)=0$,

where $\lambda(x, \delta)=\operatorname{Max}_{|\theta| \geqq \delta} f^{-1}(x)\left|f\left(x e^{i \theta}\right)\right|$.

Let $F$ be that class of functions $f(z)$ such that a $\delta(x)$ satisfying the above mentioned conditions can be defined.

Let $F_{1}$ be that class of functions $f(z) \in F$ such that $\tilde{a}(x) \in A$.

Let $r=r(n)$ be the unique root of the transcendental equation

$$
a_{1}(r)=n,
$$

which approaches $R$ às $n \rightarrow \infty$. (For proof of uniqueness see [7].)

Let $\Delta=\Delta(a)$ be the class of functions $\delta(r)$ satisfying (i), (ii) and (iii) for a given $a(x) \in A$. For $a(x) \in A$ and $m$ a fixed positive integer we define

(2) $b(x)=\operatorname{Max}\left\{a^{2 m+1} a_{2}^{-3 m-5 / 2}, a_{2}^{-3 m-3 / 2} a_{3}^{2 m} a_{4}, a_{2}^{-3 m-5 / 2} a_{3}^{2 m+2}, a_{2}^{-3 m-7 / 2} a^{2 m+2}\right\}$.

Now, choosing $\delta(x) \in \Delta(a)$ we define

$$
\begin{gathered}
\varphi_{m}(x ; \delta, a)=a_{2}(x)^{1 / 2} \operatorname{Max}\left\{\left(\delta a_{2}\right)^{-1} e^{-(1 / 2) \delta^{2} a_{2}}, \lambda(x, \delta), b(x)\right\}, \\
\varphi_{m}(x)=\varphi_{m}(x ; \delta, \tilde{a})
\end{gathered}
$$

where

(5)

$$
\begin{aligned}
\delta & =\left[\delta_{0}(x)=1 . u . b_{\delta \in \Delta(\tilde{a})} \delta(x) \text { if } \delta_{0} \in \Delta(\tilde{a})\right], \text { or } \\
& =\left[\begin{array}{l}
\delta_{k} \text { for } k \text { sufficiently large where }\left\{\delta_{k}(x)\right\} \text { is a sequence such that } \\
\delta_{k} \in \Delta(\tilde{a}) \text { and } \lim _{k \rightarrow \infty} \delta_{k}(x)=\delta_{0}(x) \notin \Delta(\tilde{a})
\end{array}\right], \\
{[x] } & =\text { greatest integer } \leqq x,
\end{aligned}
$$

$$
\begin{aligned}
& \left(\begin{array}{c}
N \\
\nu
\end{array}\right)_{k}=\frac{N !}{v_{1} ! \nu_{2} ! \cdots \nu_{k} !} \quad\left(\nu_{1}+\nu_{2}+\cdots+v_{k}=N\right), \\
& A_{N}(x)=\frac{(-1)^{N / 2}}{N !} \sum_{k=1}^{[N / 3]} \frac{1}{k !} \sum\left(\begin{array}{c}
N \\
\nu
\end{array}\right)_{k} a_{v_{1}}(x) \cdots a_{v_{k}}(x), \quad N \text { even. }
\end{aligned}
$$

REMARK. Throughout, a summation without limits will be understood to range over all sets of integers $\nu_{j} \geqq 3$ satisfying $\nu_{1}+\cdots+v_{k}=N$. By (5), we have that $A_{2}=0$ and we set $A_{0}=1$. Throughout, any $O$-term is understood for $n \rightarrow \infty$ or, equivalently, for $r \rightarrow R$. 
With these notations, Professor Grosswald [5] has proven the following theorem and corollary.

THEOREM 1. If $f(z)=\sum_{n=0}^{\infty} a_{n} z^{n} \in F$ and $r=r(n)$ is the root of (1), then, for any choice of the natural integer $m$, of $a(r) \in A$ and $\delta(r) \in \Delta(a)$, the coefficients $a_{n}$ of $f(z)$ admit the following asymptotic expansion

$$
\begin{aligned}
a_{n} & =f(r) \cdot r^{-n}\left(2 \pi^{2} a_{2}(r)\right)^{-1 / 2} \cdot\left\{\sum_{\nu=0}^{3 m}\left(2 a_{2}^{-1}(r)\right)^{\nu} \Gamma\left(\nu+\frac{1}{2}\right) A_{2 v}(r)+O\left(\varphi_{m}(r ; \delta, a)\right)\right\} \\
& =f(r) \cdot r^{-n}\left(2 \pi a_{2}(r)\right)^{-1 / 2} \cdot\left\{1+\pi^{-1 / 2} \sum_{\nu=2}^{3 m}\left(2 a_{2}^{-1}(r)\right)^{\nu} \Gamma\left(\nu+\frac{1}{2}\right) A_{2_{v}}(r)+O\left(\varphi_{m}(r ; \delta, a)\right)\right\}
\end{aligned}
$$

COROLlaRY. If $f(z) \in F_{1}$, then the error term can be replaced by $O\left(\varphi_{m}(r)\right)$ where $\varphi_{m}(r)$ is given in (4).

Let $\zeta(s)=\sum_{n=1}^{\infty}\left(1 / n^{s}\right)$ be the ordinary Riemann zeta function.

Considering the function $\xi(s)=\frac{1}{2} s(s-1) \pi^{-3 / 2} \Gamma(s / 2) \zeta(s)$, it is easy to show that from the functional equation of the Riemann zeta function, it follows that

$$
\xi(1-s)=\xi(s) .
$$

Setting $s=\frac{1}{2}+i t$, it can be shown that $\xi\left(\frac{1}{2}+i t\right)=\Xi(t)=\sum_{n=0}^{\infty} c_{n} t^{n}$ is an entire function of order 1 , of $t$.

The Riemann hypothesis states that all zeros of the Riemann zeta function that lie in the strip $0<\sigma<1$ lie on the line $\sigma=\frac{1}{2}$. It is well known, and easy to show, that the Riemann hypothesis is equivalent to all roots of the equation $\Xi(t)=0$ being real. From page 24 in [4], all roots of $\Xi(t)=0$ are real implies, if $c_{0}$ is real, that

$$
\mu_{n}=n c_{n}^{2}-(n+1) c_{n-1} c_{n+1}>0 .
$$

As Grosswald [5] shows, this condition is satisfied rather trivially. Now we set $z=-t^{2}$, thus

$$
\Xi(t)=f\left(-t^{2}\right)=f(z)=\sum_{n=0}^{\infty} \mathfrak{a}_{n} z^{n}
$$

is an entire function of order $\frac{1}{2}$. If $t_{0}$ is a real zero of $\Xi(t)$, then $z_{0}=-t_{0}^{2}$ is a negative zero of $f(z)$ and the Riemann hypothesis is equivalent to the statement that all zeros of $f(z)$ are negative. In particular they must be real, and (7) becomes

$$
D_{n}=n a_{n}^{2}-(n+1) \mathfrak{a}_{n-1} \mathfrak{a}_{n+1}>0, \quad \text { for all } n \geqq 1 \text {. }
$$

Grosswald [5] proves, using Theorem 1 and its corollary, that

$$
D_{n}=\mathfrak{a}_{n}^{2}\left(1+O\left(\log ^{-1} n\right)\right) \text {. }
$$

Clearly, (9) proves (8) for all sufficiently large values of $n$, and hence, for all sufficiently large values of $n$ this necessary condition (namely condition $C$ of $\S 1$ ), is indeed satisfied. 
III. Some definitions and theorems.

3. The general Stirling formula is well known:

$$
\log \Gamma(z)=\frac{1}{2} \log 2 \pi-z+\left(z-\frac{1}{2}\right) \log z+J(z),
$$

where

$$
J(z)=\frac{1}{\pi} \int_{0}^{\infty} \frac{z}{u^{2}+z^{2}} \log \frac{1}{1-e^{-2 \pi u}} d u=\sum_{\nu=1}^{m} \frac{B_{2 v}}{2 v(2 v-1)} z^{1-2 v}+J(m, z)
$$

$B_{v}$ are the Bernoulli numbers, and

$$
J(m, z)=\frac{(-1)^{m}}{z^{2 m+1}} \frac{1}{\pi} \int_{0}^{\infty} \frac{u^{2 m}}{1+(u / 2)^{2}} \log \frac{1}{1-e^{2 \pi u}} d u .
$$

(For a proof see $\$ 3.5$ of [1].)

We define

(11) $J_{1}(z)=z \frac{d}{d z} J\left(\frac{1+\mu_{1}}{2} z^{1 / 2}+\frac{\mu_{2}+2 \mu}{4}+6 \mu_{3}\right)=z \frac{d}{d z} J(w)$ and $J_{0}(z)=J(w)$, where

$$
w=\frac{1+\mu_{1}}{2} z^{1 / 2}+\frac{\mu_{2}+2 \mu}{4}+6 \mu_{3}
$$

and $\mu, \mu_{1}, \mu_{2}, \mu_{3}$ can be either 0 or 1 . Furthermore we define, inductively, for $\nu>1$

$$
J_{v}(z)=z \frac{d}{d z} J_{v-1}(z)
$$

Lemma. Defining the linear differential operator $D=z(d / d z)$ we have for $m$ $=1,2,3 \ldots$

$$
D^{v} J(m, w)=O\left(z^{-m-1 / 2}\right) \text { for }|\arg z|<\pi-\delta, \delta>0 .
$$

\section{Proof.}

$$
z \frac{d}{d z} J(m, w)=z \frac{d}{d z}\left\{\frac{(-1)^{m}}{w^{2 m+1}} \frac{1}{\pi} \int_{0}^{\infty} \frac{u^{2 m}}{1+\left(u^{2} / w^{2}\right)} \log \frac{1}{1-e^{-2 \pi u}} d u\right\}
$$

Because of uniform convergence we can differentiate inside the integral sign. Thus

$$
\begin{array}{r}
z \frac{d}{d z} J(m, w)=\frac{1+\mu_{1}}{4 \pi}(-1)^{m} \frac{z^{1 / 2}}{w^{2 m+2}}\left\{\frac{2}{w^{2}} \int_{0}^{\infty} \frac{u^{2 m+2}}{\left(1+\left(u^{2} / w^{2}\right)\right)^{2}} \log \frac{1}{1-e^{-2 \pi u}} d u-(2 m+1)\right. \\
\left.\cdot \int_{0}^{\infty} \frac{u^{2 m}}{1+\left(u^{2} / w^{2}\right)} \log \frac{1}{1-e^{-2 \pi u}} d u\right\}
\end{array}
$$

It is easy to show that both integrals involved in the above expression are bounded by a convergent integral. (The bound however depends on $m$.) Thus

$$
D J(m, w)=z \frac{d}{d z} J(m, w)=\frac{1+\mu_{1}}{4 \pi}(-1)^{m} \frac{z^{1 / 2}}{w^{2 m+2}} O(1)=O\left(z^{-m-1 / 2}\right) .
$$


In general $D^{k} J(m, w)$ can be expressed as a sum of $k+1$ terms. By induction on $k$ one can show that

where

$$
D^{k} J(m, w)=\sum_{i=1}^{k+1} a_{i, m}^{(k)} g_{i, m}^{(k)}(z) I_{i, m}^{(k)}(z)
$$

$a_{i, m}^{(k)}$ are constants depending on $m$ and $k$,

$I_{i, m}^{(k)}(z)$ are integrals that are bounded for $z \rightarrow \infty$,

$g_{i, m}^{(k)}(z)$ are functions of $z$ so that $\left|g_{i, m}^{(k)}(z)\right| \leqq C_{k}|z|^{-m-1 / 2}$

and

$$
C_{k} \text { is a constant depending on } k \text { only. }
$$

This completes the proof of the Lemma.

THEOREM 2. The $J_{k}(z)$ defined by (11) and $\left(11^{\prime}\right)$ satisfy, for $k=0,1,2, \ldots$

$$
J_{k}(z)=(-1)^{k} B_{2} z^{-1 / 2}\left\{2^{-\left(k+\mu_{1}\right)}-2^{1-2 \mu_{1}}\left(\frac{\mu_{2}+2 \mu}{4}+6 \mu_{3}\right) z^{-1 / 2}\right\}+D^{k} J(1, w)
$$

for $|\arg z|<\pi-\delta, \delta>0$.

Proof (by induction).

Immediate for $k=0$.

$$
J_{1}(z)=z \frac{d}{d z} J(w)=-B_{2} z^{-1 / 2}\left(\frac{2^{-1}}{1+\mu_{1}}-\frac{2\left(\left(\mu_{2}+2 \mu\right) / 4+6 \mu_{3}\right)}{\left(1+\mu_{1}\right)^{2}} z^{-1 / 2}\right)+z \frac{d}{d z} J(1, w) .
$$

Since

$$
\begin{aligned}
\frac{1}{1+\mu_{1}} & =1 & & \text { if } \mu_{1}=0, \\
& =2^{-1} & & \text { if } \mu_{1}=1,
\end{aligned}
$$

it follows that $1 /\left(1+\mu_{1}\right)=2^{-\mu_{1}}$ and the theorem holds for $k=1$.

Now to complete the proof we consider

$$
\begin{aligned}
J_{k+1}(z)= & z \frac{d}{d z} J_{k}(z) \\
= & z(-1)^{k} B_{2} \frac{d}{d z}\left\{2^{-\left(k+\mu_{1}\right)} z^{-1 / 2}-2^{1-2 \mu_{1}}\left(\left(\mu_{2}+2 \mu\right) / 4+6 \mu_{3}\right) z^{-1}\right\}+z \frac{d}{d z} D^{k} J(1, w) \\
= & (-1)^{k+1} B_{2} z^{-1 / 2}\left\{2^{-\left(k+1+\mu_{1}\right)}-2^{1-2 \mu_{1}}\left(\left(\mu_{2}+2 \mu\right) / 4+6 \mu_{3}\right) z^{-1 / 2}\right\} \\
& +D^{k+1} J(1, w) .
\end{aligned}
$$

COROLlaRY. Under the hypothesis of Theorem 2 we have

$$
\begin{aligned}
& J_{k}(z)=(-1)^{k} B_{2} z^{-1 / 2}\left\{2^{-\left(k+\mu_{1}\right)}-2^{1-2 \mu_{1}}\left(\left(\mu_{2}+2 \mu\right) / 4+6 \mu_{3}\right)\right\}+O\left(z^{-3 / 2}\right) \\
& \quad \text { for }|\arg z|<\pi-\delta, \delta>0 .
\end{aligned}
$$

Proof. Immediate from Theorem 2 and the lemma that precedes it. 
Let $r_{1}$ and $r_{2}$ be constants, we define

$$
\mathscr{J}(z)=r_{1} J\left(\frac{z^{1 / 2}}{2}+\frac{1}{4}\right)+r_{2} J\left(z^{1 / 2}+\frac{1}{2}\right) \quad \text { and } \quad \mathscr{J}_{1}(z)=z \frac{d}{d z} \mathscr{J}(z)
$$

and inductively for $\nu>1$

$$
\mathscr{J}_{v}(z)=z \frac{d}{d z} \mathscr{J}_{v-1}(z)
$$

THEOREM 3. For the $\mathscr{J}_{v}(z)(\nu=0,1,2, \ldots)$ defined by (12) we have

$$
\mathscr{J}_{v}(z)=(-1)^{v}\left(2 r_{1}+r_{2}\right) B_{2} z^{-1 / 2}\left(2^{-(v+1)}-\left(4 z^{1 / 2}\right)^{-1}\right)+O\left(z^{-3 / 2}\right)
$$

for $|\arg z|<\pi-\delta, \delta>0$.

Proof. $\mathscr{J}_{k}(z)=D^{k} \mathscr{J}(z)=r_{1} J_{k}\left(z^{1 / 2}+\frac{1}{4}\right)+r_{2} J_{k}\left(z^{1 / 2}+\frac{1}{2}\right) \quad$ where $\quad J_{k}\left(z^{1 / 2}+\frac{1}{4}\right) \quad$ and $J_{k}\left(z^{1 / 2}+\frac{1}{2}\right)$ are chosen from (11) and (11') respectively so that $\mu=\mu_{1}=\mu_{3}=0, \mu_{2}=1$ and $\mu=\mu_{1}=1, \mu_{2}=\mu_{3}=0$. Now the desired result follows from the corollary of Theorem 2.

Let $f(z)=\sum \alpha_{n} z^{n}$ be analytic, real for real $z$, positive for $z$ positive and sufficiently large (say for $z \geqq x_{0}$ ) and suppose also that $\log f(z)$ admits a representation

$$
\begin{aligned}
\log f(z)= & c_{1} z^{1 / 2} \log \left(z^{1 / 2}+c_{2}\right)+c_{3} \log \left(z-\frac{1}{4}\right) \\
& +c_{4} \log \left(z^{1 / 2}+c_{2}\right)+c_{5} z^{1 / 2}+c_{6}+\tilde{J}(z)+Q(z)
\end{aligned}
$$

where the branch of the logarithm has been selected so as to be real for $z \geqq x_{0}, c_{i}$ are fixed constants for $1 \leqq i \leqq 6\left(c_{1}>0, c_{2}>0, c_{3}=0\right.$ or $\left.1, c_{4} \geqq 0\right), \tilde{J}(z)$ may be either $\mathscr{J}(z)$ or $J(w)$, and $Q(z)$ has the property that $\left|D^{k} Q(z)\right|=O\left(x^{-3 / 2}\right)(k=0,1,2, \ldots)$ for $|\arg z|<\pi-\delta, \delta>0$ and $-\infty \leqq \operatorname{Re} Q(z)=O(\log x)$ for $\pi-\delta \leqq|\arg z| \leqq \pi$.

The following theorem shall be used in $\$ \$ 4$ and 5 .

THEOREM 4. Let $Z(s)=\sum\left(a_{n} / n^{s}\right)$ be a Dirichlet series so that $a_{n}=O\left(n^{\lambda}\right)$. If $D_{z}$ is the linear operator $z(d / d z)$, then

$$
\left|D_{z}^{k}(\log Z(s))\right|=O\left(x^{-3 / 2}\right)
$$

for $|\arg z|<\pi-\delta, \delta>0$, where $s=z^{1 / 2}+c$ and $k=0,1,2, \ldots$

Proof. Let $Q(z)=\log Z(s)$. In the range $|\arg z|<\pi-\delta$ we differentiate and let $Q^{(i)}(z)$ represent the $i$ th derivative of $Q(z)$ with respect to $s$. It follows by induction that

$$
D_{z}^{k} Q(z)=\frac{1}{2^{k}} \sum_{i=1}^{k} \alpha_{i}^{(k)} Q^{(i)}(z) z^{i / 2} \text { for } k=1,2, \ldots
$$

where $\alpha_{1}^{(k)}=\alpha_{k}=1$ and $\alpha_{i}^{(k)}=i \alpha_{i}^{(k-1)}+\alpha_{i-1}^{(k-1)}, i=2,3, \ldots, k-1$.

Clearly $Q^{(k)}(z)=\left(d^{k} / d s^{k}\right) \log Z(s)$ is a finite sum of $l$ terms, each of the form

$$
\frac{c_{j, k} Z^{\left(\alpha_{1}\right)}(s) \cdots Z^{\left(\alpha_{k}\right)}(s)}{Z^{i}(s)}
$$


for $i=1,2, \ldots, k ; j=1,2, \ldots, l$ where the $c_{j, k}$ are constants that depend on $k$. $\alpha_{i} \geqq 0$ (not necessarily distinct) satisfy $\sum_{i=1}^{k} a_{i}=k$ and $Z^{(0)}(s)$ is to be interpreted to mean 1. In the range $|\arg z|<\pi-\delta$ we have $Z(s)=\sum_{1}^{\infty}\left(a_{n} / n^{s}\right)$

$$
Z^{(1)}(s)=\sum_{1}^{\infty} \frac{a_{n}(-\log n)}{n^{s}}, \ldots, Z^{(k)}(s)=\sum_{1}^{\infty} \frac{a_{n}(-\log n)^{k}}{n^{s}} .
$$

Because $a_{n}=O\left(n^{\lambda}\right)$ it follows that

$$
Z^{\left(\alpha_{i}\right)}(s)=\frac{a_{2}(-\log 2)^{\alpha_{i}}}{2^{s}}+O\left(3^{-s}\right)
$$

Thus,

$$
\begin{aligned}
Z^{\left(\alpha_{1}\right)}(s) \cdots Z^{\left(\alpha_{k}\right)}(s) & =\frac{a_{2}^{t_{j}}(-\log 2)^{k}}{2^{s}}+O\left(3^{-s}\right), \quad 1 \leqq t_{j} \leqq k, \\
Z^{-i}(s) & =1-i a_{2} 2^{-s}+O\left(3^{-s}\right) .
\end{aligned}
$$

Thus, $Q^{(k)}(z)$ is a finite sum of $l$ terms each of the form

$$
\begin{gathered}
c_{j, k} \frac{a_{2}^{t_{j}}(-\log 2)^{k}}{2^{s}}+O\left(3^{-s}\right) ; \\
\therefore Q^{(k)}(z)=\frac{(-\log 2)^{k}}{2^{s}} \sum_{j=1}^{l} a_{2}^{t_{j}} c_{j, k}+O\left(3^{-s}\right) .
\end{gathered}
$$

Since $a_{2}^{t_{j}} \leqq a_{2}^{k}$ a constant if $a_{2}>1$ and $a_{2}^{t_{j}} \leqq 1$ if $a_{2} \leqq 1$ it follows that $Q^{(k)}=$ $d_{k} 2^{-s}(-\log 2)^{k}+O\left(3^{-s}\right)$ where $d_{k}=$ a constant,

$$
\therefore D_{z}^{k}(\log Z(s))=\frac{1}{2^{k}} \sum_{i=1}^{k} \alpha_{i}^{(k)} Q^{(i)}(z) z^{1 / 2}=O\left(z^{k / 2} 2^{-s}\right),
$$

for $k=1,2, \ldots$

For $k=0$ we have $\log Z(s)=\log \left(1+a_{2} 2^{-s}+O\left(3^{-s}\right)\right)=O\left(2^{-s}\right)$ single $a_{2}$ is a constant. Since $\delta<|\theta|<\pi-\delta$ it follows that $\cos (\theta / 2)>\delta / \pi$. Hence

$$
\begin{gathered}
\left|2^{-z^{1 / 2}}\right|=\exp \left\{\operatorname{Re}\left(-z^{1 / 2} \log 2\right)\right\}=\exp \left\{-\log 2 \cdot \operatorname{Cos}(\theta / 2) \cdot x^{1 / 2}\right\} \leqq 2^{-\delta x^{1 / 2} / \pi}, \\
\therefore\left|D_{z}^{k}(\log Z(s))\right|=O\left(x^{k / 2} 2^{-\delta x^{1 / 2} / \pi}\right)=O\left(x^{-3 / 2}\right)
\end{gathered}
$$

for $k=0,1,2, \ldots$ Q.E.D.

Now we define

$$
\begin{array}{rlr}
\delta_{i, j}=1 & \text { if } i=j \\
=0 & \text { if } i \neq j
\end{array}
$$

and

$$
a_{1}(z)=z \frac{f^{\prime}(z)}{f(z)}
$$

and inductively for $k \geqq 2$,

$$
a_{k}(z)=z a_{k-1}^{\prime}(z) .
$$


We shall now study the $a_{k}(z)$. In order to do this we state four lemmas. The first three lemmas are easily proven by induction and Lemma 4 is immediate from the three preceding lemmas.

LEMMA 1. If $D$ is the linear operator $z(d / d z)$ then

$$
D^{k}\left(c_{1} z^{1 / 2} \log \left(z^{1 / 2}+c_{2}\right)\right)=\frac{c_{1}}{2^{k}}\left(z^{1 / 2} \log e^{k}\left(z^{1 / 2}+c_{2}\right)-c_{2}+\sum_{i=1}^{k}(-1)^{i+k} \frac{b_{i}^{(k)} c_{2}^{(i+1)}}{\left(z^{1 / 2}+c_{2}\right)^{i}}\right)
$$

where the $b_{i}^{(k)}$ are constants that depend on $k$ and satisfy

$$
b_{1}^{(2 k-1)}=1, \quad b_{1}^{(2 k)}=0, \quad b_{k}^{(k)}=(k-1) ! \quad \text { for } k=1,2, \ldots
$$

and

$$
b_{i}^{(k)}=i b_{i}^{(k-1)}+(i-1) b_{i-1}^{(k-1)} \text { for } i=2,3, \ldots, k-1 .
$$

LEMMA 2. If $D$ is the linear operator $z(d / d z)$ then

$$
D^{k} \log \left(z-\frac{1}{4}\right)=\delta_{1, k}+(-1)^{k-1} \sum_{i=1}^{k} \frac{d_{i}^{(k)}}{4^{i}\left(z-\frac{1}{4}\right)^{i}}
$$

where the $d_{i}^{(k)}$ are constants that depend on $k$ and satisfy

$$
d_{1}^{(k)}=1, \quad d_{k}^{(k)}=(k-1) d_{k-1}^{(k-1)} \text { for } k=2,3, \ldots
$$

and

$$
d_{i}^{(k)}=i d_{i}^{(k-1)}+(i-1) d_{i-1}^{(k-1)} \text { for } i=2,3, \ldots, k-1 .
$$

LEMmA 3. If $D$ is the linear operator $z(d / d z)$ then

$$
D^{k} \log \left(z^{1 / 2}+c_{2}\right)=\frac{1}{2^{k}} \sum_{i=1}^{k+1} \frac{(-1)^{i+k} h_{i}^{(k+1)} c_{2}^{i-1}}{\left(z^{1 / 2}+c_{2}\right)^{i}}
$$

where the $h_{i}^{(k+1)}$ are constants which depend on $k$ and satisfy

$$
h_{1}^{(k)}=1, \quad h_{k}^{(k)}=(k-1) ! \text { for } k=2,3, \ldots
$$

and

$$
h_{i}^{(k+1)}=i h_{i}^{(k)}+(i-1) h_{i-1}^{(k)} \text { for } i=2,3, \ldots, k .
$$

LEMMA 4. For $f(z)$ satisfying (13) and any $\delta>0$ we have, uniformly in $|\arg z|<\pi-\delta$

$$
\begin{aligned}
a_{k}(z)= & \frac{c_{1}}{2^{k}} z^{1 / 2} \log e^{k}\left(z^{1 / 2}+c_{2}\right)-\frac{c_{1} c_{2}}{2^{k}}+\frac{c_{1}}{2^{k}} \sum_{i=1}^{k}(-1)^{i+k} \frac{b_{i}^{(k)} c_{2}^{i+1}}{\left(z^{1 / 2}+c_{2}\right)^{i}}+c_{3} \delta_{1, k} \\
& +(-1)^{k-1} c_{3} \sum_{i=1}^{k} \frac{d_{i}^{(k)}}{4^{i}\left(z-\frac{1}{4}\right)^{i}}+\frac{c_{4}}{2^{k}} \sum_{i=1}^{k+1} \frac{(-1)^{i+k} h_{i}^{(k+1)} c_{2}^{i-1}}{\left(z^{1 / 2}+c_{2}\right)^{i}} \\
& +\frac{c_{5}}{2^{k}} z^{1 / 2}+D^{k} \tilde{J}(z)+D^{k} Q(z)
\end{aligned}
$$

where $b_{i}^{(k)}, d_{i}^{(k)}, h_{i}^{(k+1)}$ are the constants defined in Lemmas 1, 2, and 3 respectively. 
THEOREM 5. For $f(z)$ satisfying (13) and any $\delta>0$ we have, uniformly in $|\arg z|$ $<\pi-\delta$,

$$
\begin{aligned}
a_{k}(z)= & \frac{c_{1}}{2^{k}} z^{1 / 2} \log e^{k} z^{1 / 2}+\frac{c_{5}}{2^{k}} z^{1 / 2}+c_{3} \delta_{1, k} \\
+ & \frac{(-1)^{k}}{2^{k}}\left\{\left(-\frac{c_{1} c_{2}^{2}}{2}-c_{4}\right) z^{-1 / 2}+\left[\frac{1+b_{1}^{(k)}+3 b_{2}^{(k)}}{3} c_{1} c_{2}^{3}\right.\right. \\
& \left.\left.+\left(1+h_{2}^{(k+1)}\right) c_{2} c_{4}+2^{k-2} c_{3}\right] z^{-1}\right\}+O\left(z^{-3 / 2}\right)
\end{aligned}
$$

where $\delta_{1, k}, b_{1}^{(k)}, b_{2}^{(k)}, h_{2}^{(k+1)}$ have already been defined.

Proof. From the corollary to Theorem 2, Theorem 3, and Lemma 4 we have

$$
\begin{aligned}
a_{k}(z)= & \frac{c_{1}}{2^{k}} z^{1 / 2} \log e^{k}\left(z^{1 / 2}+c_{2}\right)-\frac{c_{1} c_{2}}{2^{k}}+\delta_{1, k} c_{3} \\
& +\frac{c_{1}}{2^{k}}(-1)^{1+k}\left(\frac{b_{1}^{(k)} c_{2}^{2}}{z^{1 / 2}+c_{2}}-\frac{b_{2}^{(k)} c_{2}^{3}}{\left(z^{1 / 2}+c_{2}\right)^{2}}\right)+c_{3}(-1)^{k} \frac{d_{1}^{(k)}}{4\left(z-\frac{1}{4}\right)} \\
& +\frac{c_{4}}{2^{k}}(-1)^{1+k} \frac{h_{1}^{(k+1)}}{z^{1 / 2}+c_{2}}+\frac{c_{2} c_{4}}{2^{k}}(-1)^{k} \frac{h_{2}^{(k+1)}}{\left(z^{1 / 2}+c_{2}\right)^{2}}+\frac{c_{5}}{2^{k}} z^{1 / 2}+O\left(z^{-3 / 2}\right)
\end{aligned}
$$

Using $d_{1}^{(k)}=h_{1}^{(k+1)}=1$ and the Taylor formula we have

$$
\begin{aligned}
a_{k}(z) & =\frac{c_{1}}{2^{k}} z^{1 / 2} \log e^{k} z^{1 / 2}+\frac{c_{5}}{2^{k}} z^{1 / 2}+c_{3} \delta_{1, k}-\frac{z^{-1 / 2}}{2^{k}}\left\{c_{1} c_{2}^{2} / 2+(-1)^{k}\left(c_{1} c_{2}^{2} b_{1}^{(k)}+c_{4}\right)\right\} \\
& +\frac{z^{-1}}{2^{k}}\left\{c_{1} c_{2}^{3} / 3+(-1)^{k}\left(c_{1} c_{2}^{3} b_{1}^{(k)}+c_{2} c_{4}+2^{k-2} c_{3}+c_{1} c_{2}^{3} b_{2}^{(k)}+c_{2} c_{4} h_{2}^{(k+1)}\right)\right\}+O\left(z^{-3 / 2}\right) .
\end{aligned}
$$

If $k$ is even $b_{1}^{(k)}=0$ and if $k$ is odd $b_{1}^{(k)}=1$; thus

$$
\frac{c_{1} c_{2}^{2}}{2}+(-1)^{k}\left(c_{1} c_{2}^{2} b_{1}^{(k)}+c_{2} c_{4}\right)=(-1)^{k}\left(\frac{c_{1} c_{2}^{2}}{2}+c_{4}\right)
$$

and

$$
\frac{c_{1} c_{2}^{3}}{3}+(-1)^{k}\left(c_{1} c_{2}^{3} b_{1}^{(k)}+c_{2} c_{4}\right)=(-1)^{k}\left(\frac{1+b_{1}^{(k)}}{3} c_{1} c_{2}^{3}+c_{2} c_{4}\right) \quad \text { Q.E.D. }
$$

In order to apply Theorem 1 to $f(z)$, as given in (13), we set $|z|=x$ and choose $\delta=x^{-1 / 6} \log ^{-1 / 2} x$. Clearly $R=\infty$, and by Theorem $5, a_{k}(x)=\left(c_{1} / 2^{k+1}\right) x^{1 / 2} \log x$ $+O\left(x^{1 / 2}\right)$, for $k=1,2,3, \ldots c_{1}$ is the constant of (13) and is independent of $k$, but the $O$-term is not claimed to be uniform in $k$. Hence it follows that for every fixed $k>1$ and sufficiently large $x$ we have that $a_{k}(x)<a_{1}(x)$. Thus $f(z) \in F_{1}$, and we may choose $a(x)=\tilde{a}(x)=a_{1}(x)$. We have, as $x \rightarrow \infty$,

$$
\begin{aligned}
\delta^{2} a_{2}(x) & \sim\left(x^{1 / 3} \log ^{-1} x\right)\left(\left(c_{1} / 8\right) x^{1 / 2} \log x\right)=\left(c_{1} / 8\right) x^{1 / 6} \rightarrow \infty, \\
\delta^{3} a(x) & \sim\left(x^{-1 / 2} \log ^{-3 / 2} x\right)\left(\left(c_{1} / 4\right) x^{1 / 2} \log x\right)=\left(c_{1} / 4\right) \log ^{-1 / 2} x \rightarrow 0, \\
\delta^{2} x^{1 / 2} & =\left(x^{-1 / 3} \log ^{-1} x\right) x^{1 / 2}=x^{1 / 6} \log ^{-1} x \rightarrow \infty
\end{aligned}
$$


THEOREM 6. Let $f(z)=\sum_{n=0}^{\infty} \mathfrak{a}_{n} z^{n}$ be analytic, real for real $z$, positive for $z$ positive and sufficiently large (say, for $z \geqq x_{0}$ ) and such that $\log f(z)$ satisfies (13). If $z=x e^{i \theta}$ and $\delta=x^{-1 / 6} \log ^{-1 / 2} x$, then in the range, $\delta<|\theta|<\pi-\delta$, we have,

$$
\log \frac{|f(z)|}{f(x)}<-\frac{c_{1}}{16} x^{1 / 6}
$$

holds uniformly, for $x \rightarrow \infty$.

Proof. Using the Taylor formula (13) becomes

$$
\begin{aligned}
\log f(z)= & c_{1} z^{1 / 2} \log z^{1 / 2}+c_{1}+c_{3} \log z+\frac{c_{4}}{2} \log z \\
& +c_{5} z^{1 / 2}+c_{6}+\tilde{J}(z)+Q(z)+O\left(z^{-1 / 2}\right) .
\end{aligned}
$$

From (13) and Theorems 2 and 3 we have that

$$
\begin{aligned}
|\tilde{J}(z)|+|Q(z)| & =O\left(x^{-1 / 2}\right), \\
\log \left|e^{c_{5} z^{1 / 2}}\right| & =c_{5} x^{1 / 2} \cdot \cos \frac{\theta}{2}, \\
\log \left|z^{1 / 2 c_{1} z^{1 / 2}}\right| & =c_{1} x^{1 / 2}\left(\cos \frac{\theta}{2} \cdot \log x^{1 / 2}-\frac{\theta}{2} \sin \frac{\theta}{2}\right) .
\end{aligned}
$$

Thus

$$
\begin{aligned}
\log |f(z)|= & c_{1} x^{1 / 2}\left(\cos \frac{\theta}{2} \cdot \log x^{1 / 2}-\frac{\theta}{2} \sin \frac{\theta}{2}+\frac{c_{5}}{c_{1}} \cos \frac{\theta}{2}\right) \\
& +\left(c_{3}+\frac{c_{4}}{2}\right) \log x+c_{1}+c_{6}+O\left(x^{-1 / 2}\right) .
\end{aligned}
$$

In the range $\delta<|\theta|<\pi-\delta$ we have

$$
\frac{\delta}{2}<\frac{|\theta|}{2}<\frac{\pi}{2}, \quad \text { thus } \cos \frac{\theta}{2}<\cos \frac{\delta}{2} \text { and } \quad \frac{\theta}{2} \sin \frac{\theta}{2} \geqq \frac{\delta^{2}}{2 \pi} .
$$

Hence

$$
\log |f(z)|-\log f(x)<-c_{1}\left(1-\cos \frac{\delta}{2}\right) x^{1 / 2} \log e^{c_{5} / c_{1}} x^{1 / 2}-\frac{c_{1} \delta^{2} x^{1 / 2}}{2 \pi}+O\left(x^{-1 / 2}\right) .
$$

From the Taylor series expansion for the cosine it follows that

$$
\log \frac{|f(z)|}{f(x)}<-c_{1} \frac{\delta^{2}}{8} x^{1 / 2} \log \mathrm{e}^{c_{5} / c_{1}} x^{1 / 2}-\frac{c_{1} \delta^{2} x^{1 / 2}}{2 \pi}+O\left(\delta^{4} x^{1 / 2} \log x\right)+O\left(x^{-1 / 2}\right) .
$$

From the choice of $\delta$ we have

$$
\begin{aligned}
\log \frac{|f(z)|}{f(x)}< & -\frac{c_{1}}{8}\left(x^{1 / 6} \log ^{-1} x\right)\left(\log e^{c_{5} / c_{1}} x^{1 / 2}+4 c_{1} / \pi\right) \\
& +O\left(x^{-1 / 6} \log ^{-1} x\right)<-\frac{c_{1}}{16} x^{1 / 6} .
\end{aligned}
$$


TheOREM 7. Let $z=x e^{i \theta}$ and $\delta=x^{1 / 6} \log ^{-1} x$ then in the range $\pi-\delta \leqq|\theta| \leqq \pi$

$$
\begin{aligned}
& \mid \Gamma\left(\frac{1+\mu_{1}}{2} z^{1 / 2}\right.\left.+\frac{\mu_{2}+2 \mu}{4}+6 \mu_{3}\right) \mid \\
&<(2 \pi)^{1 / 2} \exp \left\{-\frac{\pi\left(1+\mu_{1}\right)}{4} x^{1 / 2}+O\left(x^{1 / 3} \log ^{1 / 2} x\right)\right\}
\end{aligned}
$$

where $\mu, \mu_{1}, \mu_{2}, \mu_{3}$ take only the values 0 and 1 .

Proof. We recall Stirling's formula (see [2])

$$
|\Gamma(x+i y)|=e^{-(\pi|y|) / 2}(2 \pi)^{1 / 2}(1+O(1 /|y|)) .
$$

Thus, letting

$$
w=\frac{1+\mu_{1}}{2} z^{1 / 2}+\frac{\mu_{2}+2 \mu}{4}+6 \mu_{3}
$$

we have

$$
\begin{aligned}
|\Gamma(w)|= & (2 \pi)^{1 / 2} \exp \left\{-\frac{\pi}{2}\left|\frac{1+\mu_{1}}{2} x^{1 / 2} \sin \frac{\theta}{2}\right|\right\} \\
& \cdot\left|\frac{1+\mu_{1}}{2} x^{1 / 2} \sin \frac{\theta}{2}\right|^{\psi(x, \theta)} \cdot\left(1+O\left(\left|\frac{1+\mu_{1}}{2} x^{1 / 2} \sin \frac{\theta}{2}\right|^{-1}\right)\right)
\end{aligned}
$$

where

$$
\psi(x, \theta)=\frac{1+\mu_{1}}{2} x^{1 / 2} \cos \frac{\theta}{2}+\frac{\mu_{2}+2 \mu+24 \mu_{3}-2}{4} .
$$

In the range, $\pi-\delta \leqq|\theta| \leqq \pi$, since $|\theta / 2|$ lies completely in the first and fourth quadrants, we have $0<\cos (\theta / 2)<(\delta / 2)$ and $1>|\sin (\theta / 2)|>\sin (|\theta| / 2)>1-\frac{1}{2}(\delta / 2)^{2}$. Because $\mu_{1}=0$ or 1 it is clear that

$$
\frac{1}{2}-\frac{\delta^{2}}{16}<\left|\frac{1}{2} \sin \frac{\theta}{2}\right| \leqq\left|\frac{1}{2}\left(1+\mu_{1}\right) \sin \frac{\theta}{2}\right|<\left|\sin \frac{\theta}{2}\right|<1
$$

and

$$
O\left(\left|\frac{1+\mu_{1}}{2} x^{1 / 2} \sin \frac{\theta}{2}\right|^{-1}\right)=O\left(x^{-1 / 2}\right)
$$

Thus

$$
|\Gamma(w)|<(2 \pi)^{1 / 2} \exp \left\{-\frac{\pi\left(1+\mu_{1}\right)}{4} x^{1 / 2}\left(1-\frac{\delta^{2}}{8}\right)\right\}\left(x^{1 / 2}\right)^{\psi(x, \delta)}\left(1+O\left(x^{-1 / 2}\right)\right) . \quad \text { Q.E.D. }
$$

REMARK. Throughout the remainder of this paper $\delta=\delta(x)$ will always be understood to have the value $\delta(x)=x^{-1 / 6} \log ^{-1 / 2} x$.

We shall now verify that if a function $f(z)=\sum \alpha_{n} z^{n}$ admits the representation (13) and also satisfies the hypothesis of Theorem 1, then its coefficients $\alpha_{n}$ satisfy (9) with $D_{n}$ defined by (8). For the remainder of this section we will assume that (17) holds in the sector $|\theta|>\delta$. For the particular $f(z)$ 's that we deal with in the following sections Theorem 7 will be used in order to verify this result. First we replace $x$ 
by the root $r=r(n)$, of the transcendental equation (1). From (1) and (15) with $k=1$ it follows that

$$
n=\frac{c_{1}}{2} r^{1 / 2} \log e r^{1 / 2}+\frac{c_{5}}{2} r^{1 / 2}+c_{3}+\frac{r^{-1 / 2}}{2}\left(\left(c_{1} c_{2}^{2} / 2\right)+c_{4}\right)
$$

$$
-\frac{r^{-1}}{2}\left(\left(2 c_{1} c_{2}^{3} / 3\right)+2 c_{2} c_{4}+\left(c_{3} / 2\right)\right) r^{-1}+O\left(r^{-3 / 2}\right)
$$

A rearrangement yields

$$
\begin{aligned}
r^{1 / 2} \log e^{1+c_{5} / c_{1}} r^{1 / 2}= & \frac{2}{c_{1}}\left(n-c_{3}\right)-\left(\left(c_{2}^{2} / 2\right)+\left(c_{4} / c_{1}\right)\right) r^{-1 / 2} \\
& +\left(\left(2 c_{2}^{3} / 3\right)+\left(2 c_{2} c_{4} / c_{1}\right)+\left(c_{3} / 2 c_{1}\right)\right) r^{-1}+O\left(r^{-3 / 2}\right)
\end{aligned}
$$

Some consequences of $(20)$ are

THEOREM 8. If $r$ is defined as the root of (1), then

(i) $\left(r^{1 / 2} \log e c_{2} r^{1 / 2}\right)^{-t}=O\left(n^{-t}\right)$ for every $t>0$,

(ii) $\log r-2 \log n \sim-2 \log \log n$,

(iii) $\frac{c_{1}}{2} r^{1 / 2}=n \log ^{-1} n(1+O((\log \log n / \log n)))$.

Proof. (i) is immediate from (20) and (iii) follows from (19) and (ii). In order to prove (ii) we first rearrange (20) to obtain

$$
\begin{aligned}
& r^{1 / 2} \log e^{1+c_{5} / c_{1}} r^{1 / 2}=\frac{2 n}{c_{1}}\left\{1+\left(c_{3} / n\right)-\left(\left(c_{1} c_{2}^{2} / 4 n\right)+\left(c_{4} / 2 n\right)\right) r^{-1 / 2}\right. \\
&\left.+\left(\left(c_{1} c_{2}^{3} / 3 n\right)+\left(c_{2} c_{4} / n\right)+\left(c_{3} / 4 n\right)\right) r^{-1}\right\}+O\left(r^{-3 / 2}\right) .
\end{aligned}
$$

Taking logs we get

$$
\frac{1}{2} \log r+\log \log r-\log 2+O((1 / \log r))=\log n+\log \left(2 / c_{1}\right)+O(1 / n)
$$

since $\log \log r=\log o(\log r)$ it follows that $\frac{1}{2} \log r \sim \log n$ and $\log \log r \sim \log \log n$ and the desired result follows. Q.E.D.

We choose $m=2$ and we apply Theorem 1, getting

$$
\alpha_{n}=r^{-n}\left(2 \pi a_{2}(r)\right)^{-1 / 2} f(r)\{1+S\}
$$

where

$$
S=\pi^{-1 / 2} \sum_{\nu=2}^{6}\left(2 a_{2}^{-1}(r)\right)^{\nu} \Gamma\left(\nu+\frac{1}{2}\right) A_{2 v}+O\left(\varphi_{2}(r)\right)
$$

and $\varphi_{2}(r)$ and $A_{2 v}$ are given by (4) and (5) respectively.

Throughout the rest of this section we let $e^{2+\left(c_{5} / c_{1}\right)}=g$.

THEOREM 9. Let $f$ be a function satisfying the hypothesis of Theorem 1 and also (13). If $S$ is defined by (23), then

$$
S=-(24 n)^{-1}+O\left(n^{-1} \log ^{-1} n\right) .
$$


Proof. Under the hypothesis of the theorem we have

(i) $\varphi_{2}(r)=O\left(n^{-2}\right)$,

(ii) $\sum_{\nu=4}^{6}\left(2 a_{2}^{-1}\right)^{\nu} \Gamma\left(\nu+\frac{1}{2}\right) A_{2 v}(r)=O\left(n^{-2}\right)$,

(iii) $\frac{a_{4}(r)}{a_{2}^{2}(r)}=\left(c_{1} r^{1 / 2} \log g r^{1 / 2}\right)^{-1}\left(1+2 \log ^{-1} g r^{1 / 2}+O\left(n^{-2} \log n\right)\right)$,

(iv) $\frac{a_{3}^{2}(r)}{a_{2}^{3}(r)}=\left(c_{1} r^{1 / 2} \log r^{1 / 2}\right)^{-1}\left(1+2 \log ^{-1} g r^{1 / 2}+\log ^{-2} g r^{1 / 2}+O\left(n^{-2} \log n\right)\right)$.

(i) is immediate from (15), (16) and the definitions of $\delta, \lambda(r), b(r)$ and $\varphi_{2}(r)$.

(ii) is immediate from (5), (15), and (21).

(iii) and (iv) are immediate from (15), (21) and the definitions of $b_{i}, h_{i}$. Now the theorem follows, after much computation, from (20), (21) and (i), (ii), (iii) and (iv) above. Q.E.D.

Returning to (22) we see that $\alpha_{n}$ becomes

$$
\alpha_{n}=r^{-n}\left(2 \pi a_{2}(r)\right)^{-1 / 2} f(r)\left(1-(24 n)^{-1}+O\left(n^{-1} \log ^{-1} n\right)\right) .
$$

The next theorem considers $D_{n}$ of the analytic function $f(z)=\sum \alpha_{n} z^{n}$ satisfying (13) and Theorem 1. Not only is (8) satisfied but the stronger (9) is also satisfied for all sufficiently large integers $n$. The proof of the next theorem is omitted because it essentially follows Professor Grosswald's paper [5].

THEOREM 10. If $f(z)$ satisfies the conditions of Theorem 1 and (13) then

$$
D_{n}=\alpha_{n}^{2}\left(1+O\left(\log ^{-1} n\right)\right) \text {. }
$$

IV. Examples of functions in $F_{2}$ which satisfy condition $\mathrm{C}$.

(1) Dirichlet L-functions. Let $m$ be a positive integer and let $\chi$ be a real, nonprincipal, primitive character $\bmod m$. The $L$-series associated with $m$ is defined to be

$$
L(s, \chi)=\sum_{n=1}^{\infty} \chi(n) n^{-s}
$$

We define

(27) $\xi(s, \chi)=\left(\frac{\pi}{m}\right)^{-(s+\mu) / 2} \dot{\Gamma}\left(\frac{s+\mu}{2}\right) L(s, \chi) \quad$ where $\mu= \begin{cases}0 & \text { if } \chi(-1)=1, \\ 1 & \text { if } \chi(-1)=-1 .\end{cases}$

It is well known that $\xi(s, \chi)$ is an entire function of $s$ of order 1 satisfying, for our choice of $\chi$, the functional equation

$$
\xi(s, \chi)=\xi(1-s, \chi) .
$$

Since $\xi\left(\frac{1}{2}+i t, \chi\right)=\xi\left(\frac{1}{2}-i t, \chi\right)$, we have symmetry about the line $\sigma=\frac{1}{2}$ and we define

$$
\Xi(z, \chi)=\xi\left(\frac{1}{2}+i z, \chi\right)=\left(\frac{\pi}{m}\right)^{-(1+2 \mu) / 4-(i z / 2)} \Gamma\left(\frac{1+2 \mu}{4}+\frac{i z}{2}\right) L\left(\frac{1}{2}+i z, \chi\right) .
$$


Hence all zeros of $\Xi(z, \chi)$ are real if and only if all zeros of $L$ lie on the line $\sigma=\frac{1}{2}+i z$.

The Riemann hypothesis for the Dirichlet $L$-function claims that all zeros of $L(s, \chi)$ that lie in the strip $0<\sigma<1$ where $\sigma=\operatorname{Re} s$ lie on the line $\sigma=\frac{1}{2}$, and this will be the case if and only if all zeros of $\Xi(z)$ are real. Let

$$
f(z, \chi)=\Xi\left(i z^{1 / 2}, \chi\right)=\xi\left(\frac{1}{2}-z^{1 / 2}, \chi\right)=\xi\left(\frac{1}{2}+z^{1 / 2}, \chi\right)=\sum_{n=0}^{\infty} \alpha_{n} z^{n} .
$$

Since $\xi(s, \chi)$ is an entire function of order 1 (a proof of this can be found in [10]), it follows that $f(z, \chi)$ is an entire function of order $\frac{1}{2}$. If $t_{0}$ is a real zero of $\Xi(t, \chi)$ then $z=-t_{0}^{2}$ is a negative zero of $f(z, \chi)$. Hence, the Riemann hypothesis for $L(s, \chi)$ is equivalent to the statement that all the zeros of $f(z, \chi)$ are negative. Since $f(z, \chi)$ is an entire function of $z$, it follows from page 24 of [4] that (8) must hold for all $n \geqq 1$.

Throughout the rest of this paper, when we say that an entire function is associated with another function, we shall mean that the entire function stands in the same relation to this other function as $f(z)$ stands in relation to $\zeta(s)$ in [5] or as $f(z, \chi)$ stands in relation to $L(s, \chi)$ in this paper. By using the results of $\S I I I$, after showing that $f(z, \chi)$ satisfies the hypothesis of Theorem 1 , we prove

THEOREM 11. If $L(s, \chi)$ is a Dirichlet L-function with $\chi$ a real, nonprincipal, primitive character and if $f(z, \chi)=\sum_{n=0}^{\infty} \alpha_{n} z^{n}$ is the entire function of order $\frac{1}{2}$ that is associated with it, then the coefficients of $f(z)$ satisfy (9).

Proof. Since $f(z, \chi)=\xi\left(\frac{1}{2}+z^{1 / 2}, \chi\right)$, we apply (29) and take the logarithm of each side, hence

$$
\log f(z, \chi)=-\left(\frac{1+2 \mu}{4}+\frac{z^{1 / 2}}{2}\right) \log \frac{\pi}{m}+\log \Gamma\left(\frac{1+2 \mu}{4}+\frac{z^{1 / 2}}{2}\right)+\log L\left(\frac{1}{2}+z^{1 / 2}, \chi\right) .
$$

Now we apply (10) and it follows that

$$
\begin{aligned}
\log f(z, \chi)= & -\left(\frac{1+2 \mu}{4}+\frac{z^{1 / 2}}{2}\right) \log \frac{\pi}{m}+\frac{1}{2} \log 2 \pi-\frac{1+2 \mu}{4}-\frac{z^{1 / 2}}{2} \\
& +\left(\frac{z^{1 / 2}}{2}+\frac{2 \mu-1}{4}\right) \log \left(\frac{1+2 \mu}{4}+\frac{z^{1 / 2}}{2}\right)+J\left(\frac{1+2 \mu}{4}+\frac{z^{1 / 2}}{2}\right)+\log L\left(\frac{1}{2}+z^{1 / 2}, \chi\right) \\
= & \frac{z^{1 / 2}}{2} \log \left(\frac{z^{1 / 2}}{2}+\frac{2 \mu+1}{2}\right)+\frac{2 \mu-1}{4} \log \left(z^{1 / 2}+\frac{2 \mu+1}{2}\right) \\
& +\left(\frac{1}{2} \log \frac{m}{2 \pi e}\right) z^{1 / 2}+c_{6}+\tilde{J}(z)+Q(z, \chi),
\end{aligned}
$$

where $Q(z, \chi)=\log L\left(\frac{1}{2}+z^{1 / 2}, \chi\right)$.

$\tilde{J}(z)=J\left((1+2 \mu) / 2+z^{1 / 2} / 2\right)\left(\mu_{1}=\mu_{3}=0, \mu_{2}=1\right.$ and $\mu$ may be either 0 or 1$)$ $c_{1}=\frac{1}{2}, \quad c_{2}=(2 \mu+1) / 2, \quad c_{3}=0, \quad c_{4}=(2 \mu+1) / 4, \quad c_{5}=\frac{1}{2} \log (m / 2 \pi e), \quad c_{6}=\frac{1}{2} \log 2 \pi-$ $((2 \mu-1) / 4) \log 2-((2 \mu+1) / 4) \log (\pi e / m)$. The coefficients of $L(s, \chi)$ are $|\chi(n)| \leqq 1$, 
so that $Q(z, \chi)$ clearly satisfies the condition $a_{n}=O\left(n^{\lambda}\right)$ of Theorem 4 with $\lambda=0$. Considering the range $\pi-\delta \leqq|\theta| \leqq \pi$ we have that

$$
0 \leqq\left|L\left(z^{1 / 2}+\frac{1}{2}, \chi\right)\right| \leqq \sum_{n=1}^{\infty} n^{-\operatorname{Re}\left(z^{1 / 2}+1\right)}=\zeta\left(x^{1 / 2} \cos (\theta / 2)+\frac{1}{2}\right)=1+o(1) .
$$

Hence

$$
-\infty \leqq \operatorname{Re} Q(z, \chi)=O(\log x) .
$$

Thus $f(z, \chi)$ satisfies (13) and also the hypothesis of Theorem 6. Hence (17) of Theorem 6 becomes

$$
f^{-1}(x, \chi) \cdot|f(z, \chi)|<\exp \left\{-x^{1 / 6} / 32\right\}=\lambda(x)
$$

uniformly in $\delta \leqq|\theta| \leqq \pi-\delta$, for $x \rightarrow \infty$. In order to complete the verification that $f(z, \chi) \in F$, we must show that (30) holds also for $\pi-\delta \leqq|\theta| \leqq \pi$. Once this is verified (30) will hold as required by Theorem 1 , for all values of $\theta$, except $|\theta| \leqq \delta$. We choose $\mu_{1}=\mu_{3}=0$ and $\mu_{2}=1$, and we apply Theorem 7. Then (18) becomes

$$
\left|\Gamma\left(\frac{1}{2} z^{1 / 2}+(1+2 \mu) / 4\right)\right|<\sqrt{2 \pi} \exp \left\{-\pi / 4+O\left(x^{1 / 3} \log ^{1 / 2} x\right)\right\} .
$$

Because in this range $-\infty \leqq \operatorname{Re} Q(z, x)=O(\log x)$,

$$
-\infty \leqq \log \left|f\left(x e^{i \theta}, \chi\right)\right|<-\frac{\pi}{5} x^{1 / 2}
$$

and

$$
\log \frac{\left|f\left(x e^{i \theta}, \chi\right)\right|}{f(x, \chi)}<-x^{1 / 2}\left(\frac{1}{2} \log \frac{x^{1 / 2} m}{2 \pi e}+\frac{\pi}{5}\right)<-\frac{1}{32} x^{1 / 6}=\log \lambda(x)
$$

holds uniformly also for $\pi-\delta \leqq|\theta| \leqq \pi$. This completes the proof that $f(z, \chi) \in F$, and since $\tilde{a}(x, \chi)=a_{1}(x, \chi)$ it follows that $f(z, \chi) \in F_{1}$. Now Theorem 11 follows from (25) and Theorems 9 and 10. Q.E.D.

(2) Ramanujan's arithmetical function. The function $\tau(n)$, defined in $\S I$, is considered by Ramanujan in his memoir [11]. The associated Dirichlet series

$$
Z(s, \tau)=\sum_{n=1}^{\infty} \frac{\tau(n)}{n^{s}}
$$

is absolutely convergent and $Z(s, \tau)$ is regular for $\sigma>13 / 2$. Wilton [12] proves that $Z(s, \tau)$ is an entire function satisfying

$$
(2 \pi)^{-s} \Gamma(s) Z(s, \tau)=(2 \pi)^{s-12} \Gamma(12-s) Z(12-s, \tau)
$$

and that it has simple zeros at $s=0,-1,-2, \ldots$ We define

$$
\xi(s, \tau)=(2 \pi)^{-s} \Gamma(s) Z(s, \tau) .
$$


Since the poles of $\Gamma(s)$ are cancelled by the zeros of $Z(s, \tau)$, it follows that $\xi(s, \tau)$ is an entire function of $s$. Thus the functional equation (32) can be written as

$$
\xi(12-s, \tau)=\xi(s, \tau) .
$$

Since $\xi(6+i t, \tau)=\xi(6-i t, \tau)$, we have symmetry about the line $\sigma=6$ and we define

$$
\Xi(z, \tau)=\xi(6+i t, \tau)=(2 \pi)^{-6-i z} \Gamma(6+i z) Z(6+i z, \tau) .
$$

Hence, all zeros of $\Xi(z, \tau)$ are real if and only if all zeros of $Z(s, \tau)$ lie on the line $\sigma=6+i z$. Using a variation of the method by which Hardy first proved that the Riemann zeta function has an infinite number of roots on the critical line $\sigma=\frac{1}{2}$, Wilton [12], proves that $Z(s, \tau)$ has an infinite number of roots on the line $\sigma=6$.

The Riemann hypothesis for $Z(s, \tau)$ claims that all zeros of $Z(s, \tau)$ that lie in the critical strip $11 / 2<\sigma<13 / 2$, where $\sigma=\operatorname{Re} s$, lie on the line $\sigma=6$, and this will be the case if all zeros of $\Xi(z, \tau)$ are real. Suppose

$$
f(z, \tau)=\Xi\left(i z^{1 / 2}, \tau\right)=\xi\left(6-z^{1 / 2}, \tau\right)=\xi\left(6+z^{1 / 2}, \tau\right)=\sum_{n=0}^{\infty} \alpha_{n} z^{n} .
$$

Since $\xi(s, \tau)$ is an entire function of order 1 , we have that $f(z, \tau)$ is an entire function of order $\frac{1}{2}$. If $t_{0}$ is a real zero of $\Xi(t, \tau)$ then $z=-t_{0}^{2}$ is a negative zero of $f(z, \tau)$. Hence the Riemann hypothesis for $Z(s, \tau)$ is equivalent to the statement that all the zeros of $f(z, \tau)$ are negative. Once again, it follows from [4] that (8) must hold for all $n \geqq 1$.

By following the procedure outlined in the proof of Theorem 11 it can be shown that

THEOREM 12. Let $Z(s, \tau)$ be given by (31). If $f(z, \tau)=\Xi\left(i z^{1 / 2}, \tau\right)=\sum_{n=0}^{\infty} \alpha_{n} z^{n}$, is the entire function that is associated with $Z(s, \tau)$, then the coefficients of $f(z, \tau)$ satisfy (9).

(3) Dedekind zeta function. Let $K$ be an algebraic number field of degree $N$. The Dedekind zeta function is

$$
\zeta(s, K)=\sum_{\mathfrak{U}}(N \mathfrak{U})^{-s},
$$

where the summation is over all nonzero integral ideals in the field $K$, and $N \mathfrak{A}$ is the norm of the ideal $\mathfrak{A}$ in $K$, and the sum converges for $\sigma>1$. We let

$r_{1}=$ number of real conjugates of $K$,

$2 r_{2}=$ number of imaginary conjugates of $K$,

$\Delta=$ discriminant.

It can be shown that $\zeta(s, K)$ can be continued analytically throughout the entire plane and that it satisfies a functional equation which can be written as

$$
\xi_{1}(s, K)=\xi_{1}(1-s, K)
$$

where

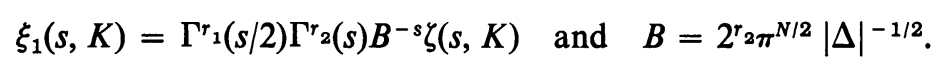


Since $\zeta(s, K)$ has simple poles at $s=0$ and $s=1$, so has $\xi_{1}(s, K)$. Thus we define

$$
\xi(s, K)=\frac{1}{2} s(s-1) \xi_{1}(s, K) .
$$

From the properties of $\zeta(s, K)$ it follows that $\xi(s, K)$ is an entire function of order 1 satisfying

$$
\xi(s, K)=\xi(1-s, K)
$$

Because of the symmetry about the line $\sigma=\frac{1}{2}$ we define

$$
\Xi(z, K)=\xi\left(\frac{1}{2}+i z, K\right) .
$$

It can be shown that $\Xi(z, K)$ is an even entire function of order 1. The Riemann hypothesis for $\zeta(s, K)$ claims that all the zeros of $\zeta(s, K)$ which lie in the strip $0<\sigma<1$, where $\sigma=\operatorname{Re}(s)$, lie on the line $\sigma=\frac{1}{2}+i t(t$ real). This will occur if and only if all zeros of $\Xi(z, K)$ are real. We define $f(z, K)=\Xi\left(i z^{1 / 2}, K\right)=\xi\left(\frac{1}{2}-z^{1 / 2}, K\right)$ $=\xi\left(\frac{1}{2}+z^{1 / 2}, K\right)=\sum_{n=0}^{\infty} \alpha_{n} z^{n}$. Clearly

$$
f(z, K)=\frac{1}{2}\left(z-\frac{1}{4}\right) \Gamma^{r}\left(\frac{1}{4}+z^{1 / 2} / 2\right) \Gamma^{r}\left(\frac{1}{2}+z^{1 / 2}\right) B^{-1 / 2-z^{1 / 2}} \zeta\left(\frac{1}{2}+z^{1 / 2}, K\right) .
$$

Since $\xi(s, K)$ is an entire function of order 1 , it follows that $f(z, K)$ is an entire function of $z$ of order $\frac{1}{2}$. If $t_{0}$ is a real zero of $\Xi(t, K)$ then $z=-t_{0}^{2}$ is a negative zero of $f(z, K)$. It follows that the Riemann hypothesis for $\zeta(s, K)$ is equivalent to the statement that all the zeros of $f(z, K)$ are negative.

Once again, following the general procedure outlined in the proof of Theorem 11 it can be shown that

THEOREM 13. Let $\zeta(s, K)$ be given by (36). If $f(z, K)=\Xi\left(i z^{1 / 2}, K\right)=\sum_{n=0}^{\infty} \alpha_{n} z^{n}$ is the entire function that is associated with $\zeta(s, K)$, then its coefficients satisfy (9).

\section{Other examples.}

(1) Epstein zeta function. We now consider a special case of the Epstein zeta function defined by

$$
\mathrm{Z}(s, Q)=\sum_{m} \sum_{n}^{\prime}\{Q(m, n)\}^{-s}
$$

where the dash indicates that the summation is over all integer values of $m$ and $n$ except $m=n=0, Q(m, n)=m^{2}+d n^{2}, \Delta=4 d$ and the number of classes of quadratic forms of discriminant $-\Delta$ is greater than one. Mordell [18] shows that $Z(s, Q)$ $=\left(2 \pi / \Delta^{1 / 2}\right)^{2 s-1}(\Gamma(1-s) / \Gamma(s)) Z(1-s, Q)$ is a meromorphic function with a single pole at $s=1$ and is analytic and regular for $s \neq 1$. Thus, defining

$$
\xi(s, Q)=\frac{1}{2} s(s-1)\left(2 \pi / \Delta^{1 / 2}\right)^{-s} \Gamma(s) Z(s, Q),
$$

we have

$$
\xi(1-s, Q)=\xi(s, Q)
$$


Thus $\xi(s, Q)$ is an entire function of order 1 that is symmetrical about the line $\sigma=\frac{1}{2}$ and we define

$$
\Xi(z, Q)=\xi\left(\frac{1}{2}+i z, Q\right) .
$$

It can be shown that $\Xi(z, Q)$ is an even entire function of order 1 . The Riemann hypothesis for $\mathrm{Z}(s, Q)$, which as we pointed out earlier is false, claims that all the zeros of $\mathrm{Z}(s, Q)$ which lie in the strip $0<\sigma<1(\sigma=\operatorname{Re} s)$ lie on the line $\sigma=\frac{1}{2}+i t$. This will occur if and only if all zeros of $\Xi(z, Q)$ are real. We define

$$
f(z, Q)=\Xi\left(i z^{1 / 2}, Q\right)=\xi\left(\frac{1}{2}-z^{1 / 2}, Q\right)=\xi\left(\frac{1}{2}+z^{1 / 2}, Q\right)=\sum_{n=0}^{\infty} \alpha_{n} z^{n}
$$

Clearly

$$
f(z, Q)=\frac{1}{2}\left(z-\frac{1}{4}\right)\left(2 \pi / \Delta^{1 / 2}\right)^{-1 / 2-z^{1 / 2}} \Gamma\left(\frac{1}{2}+z^{1 / 2}\right) Z\left(\frac{1}{2}+z^{1 / 2}, Q\right) .
$$

Since $\xi(z, Q)$ is an entire function of order 1 , it follows that $f(z, Q)$ is an entire function of order $\frac{1}{2}$. If $t_{0}$ is a real zero of $\Xi(t, Q)$, then $z=-t_{0}^{2}$ is a negative zero of $f(z, Q)$. Thus the Riemann hypothesis for $\mathrm{Z}(s, Q)$ is equivalent to the statement that all the zeros of $f(z, Q)$ are negative.

Once again following the general procedure outlined in the proof of Theorem 11, we have

THEOREM 14. Let $\mathrm{Z}(s, Q)$ be given by (41). If $f(z, Q)=\Xi\left(i z^{1 / 2}, Q\right)=\sum_{n=0}^{\infty} \alpha_{n} z^{n}$ is the entire function that is associated with $\mathrm{Z}(s, Q)$, then its coefficients satisfy (9).

(2) Class $F$ function for which condition $\mathbf{C}$ does not hold. Hayman [7] proves $e^{z}=\sum_{n=0}^{\infty}\left(z^{n} / n !\right) \in F$. For this function $\alpha_{n}=1 / n$ ! and $D_{n}=n \alpha_{n}^{2}-(n+1) \alpha_{n-1} \alpha_{n+1}=0$. Calling this function $\tilde{f}(z)$ one may work backwards and construct a function $\tilde{\zeta}(s)$ that stands in the same relation to $f(z)$ as $\zeta(s)$ stands in relation to the function $f(z)$ of [5]. (Or, equivalently, as $L(s, \chi)$ stands in relation to $f(z, \chi)$ in this paper.) It is easy to show that $\tilde{\zeta}(s) \in F_{1}$, and one finds

$$
\tilde{\zeta}(s)=\frac{2 \pi^{s / 2}}{s(s-1) \Gamma(s / 2)} e^{(s-1 / 2)^{2}}
$$

a meromorphic function with only one pole at $s=1$ with residue $2 e^{1 / 4}$ there. This function has zeros at $s=-2,-4, \ldots$, and it has no complex zeros.

\section{BIBLIOGRAPHY}

1. L. Ahlfors, Complex analysis, McGraw-Hill, New York, 1953.

2. R. Ayoub, Introduction to the analytic theory of numbers, Math. Surveys, No. 10, Amer. Math. Soc., Providence, R. I., 1963.

3. R. Bateman and E. Grosswald, On Epstein's zeta function, Acta Arith. 9 (1964), 365-373.

4. R. Boas, Entire functions, Academic Press, New York, 1954.

5. E. Grosswald, Generalization of a formula of Hayman and its application to the study of Riemann's zeta functions, Illinois J. Math. 10 (1966), 9-23. 
6. J. Grommer, Ganze transzendente Funktionen mit lauter reellen Nullstellen, J. Reine Angew. Math. 144 (1914), 114-165.

7. W. Hayman, A generalization of Stirling's formula, J. Reine Angew. Math. 196 (1956), 57-95.

8. L. Mordell, The zeta functions arising from quadratic forms and their functional equations, Quart. J. Math. Oxford Ser. 1 (1930), 77-101.

9. G. Pólya, Uber die Algebraisch-Funktionen theoretischen von J. L. W. V. Jensen, Mat.-Fys. Medd. Danske Vid. Selsk. 7 (1927), 1-33; p. 16.

10. K. Pracher, Primzahlverteilung, Springer-Verlag, Berlin, 1927.

11. S. Ramanujan, On certain arithmetical functions, Trans. Cambridge Philos. Soc. 22 (1916), 159-184. (Collected Papers No. 22.)

12. J. R. Wilton, A Note on Ramanujan's arithmetical function $\tau(n)$, Proc. Cambridge Philos. Soc. 25 (1929), 121-129.

UNIVERSITY OF CALIFoRnia,

Los ANgeles, California 